\title{
Path integral Monte Carlo calculations of helium and hydrogen-helium plasma thermodynamics and of the deuterium shock Hugoniot
}

\author{
P R Levashov ${ }^{1}$, V S Filinov ${ }^{1}$, M Bonitz ${ }^{2}$ and V E Fortov ${ }^{1}$ \\ ${ }^{1}$ Institute for High Energy Densities, RAS, Izhorskaya 13/19, Moscow 125412, Russia \\ E-mail: pasha@ihed.ras.ru \\ ${ }^{2}$ Christian-Albrechts-Universität zu Kiel, Institut für Theoretische Physik und \\ Astrophysik, Leibnizstr. 15, 24098 Kiel, Germany
}

\begin{abstract}
In this work we calculate the thermodynamic properties of hydrogenhelium plasmas with different mass fractions of helium by the direct path integral Monte Carlo method. To avoid unphysical approximations we use the path integral representation of the density matrix. We pay special attention to the region of weak coupling and degeneracy and compare the results of simulation with a model based on the chemical picture. Further with the help of calculated deuterium isochors we compute the shock Hugoniot of deuterium. We analyze our results in comparison with recent experimental and calculated data on the deuterium Hugoniot.

PACS numbers: $52.25 . \mathrm{Kn}, 52.27 . \mathrm{Gr}, 31.15 . \mathrm{Kb}, 62.50 .+\mathrm{p}$
\end{abstract}

\section{Introduction}

Hydrogen and helium are the most abundant elements in the Universe, therefore thermodynamic properties of hydrogen and helium plasmas are widely required for many astrophysical problems [1-4]. In particular, the investigation of the giant planets Jupiter and Saturn, and to a lesser extent brown dwarfs demands the thermodynamic information for hydrogen and helium in the approximate range of temperatures $10^{3}<T<10^{5} \mathrm{~K}$ and mass densities $0.01<\rho<100 \mathrm{~g} / \mathrm{cm}^{3}$. This region is characterized by coupling effects and chemical reactions caused by partial pressure dissociation and ionization $[5,6]$; these effects considerably complicate an equation of state (EOS) calculation. Moreover, in the same range of parameters the so-called plasma phase transition (PPT) has been predicted by many authors $[1,5-8]$. However the application of the chemical picture $[5,6]$ at densities corresponding to pressure ionization is questionable. Therefore there is a great interest in direct first-principle numerical simulations of strongly coupled degenerate systems which avoid difficulties of conventional theories. 
In this work we use the direct path integral Monte Carlo method (DPIMC) to calculate thermodynamic properties of hydrogen-helium plasma with different mass fractions of helium. This method is well established theoretically and allows the treatment of quantum and exchange effects without any approximations using only fundamental physical constants. We compare the results of our simulation with the EOS model based on the chemical picture [1,2]. We also use the DPIMC method to compute the deuterium Hugoniot. We compare our simulation results with recent experimental and theoretical works and analyze the modern state of the problem.

\section{Simulation method and results for hydrogen-helium plasma}

The details of our computational scheme can be found elsewhere [9-12]. Modern supercomputers allow us to simulate about 100 quantum particles in a Monte Carlo cell at a given temperature and volume. The DPIMC has no limitations on coupling parameter and can be applied at significant degeneracy of the system (with degeneracy parameter values as high as 300) [10]. Earlier the method was thoroughly tested by simulating different properties of ideal and interacting degenerate plasmas $[13,14]$. In particular, we investigated temperature and pressure dissociation and ionization $a b$ initio; we also observed the effect of proton ordering at very high densities and the formation of a Coulomb crystal of protons [13].

In this section we calculate thermodynamic properties of hydrogen-helium mixtures at relatively low coupling and degeneracy parameters and compare our results with a well-known chemical picture model used mostly in astrophysics [1,2]. This model includes classical statistics for molecules and ions and Fermi-Dirac statistics for the electrons. It takes into account many physical effects including a number of subtle "second-order" phenomena. We calculated thermodynamic properties of hydrogenhelium mixtures with a composition corresponding to that of the outer layers of the Jovian atmosphere. During the mission of the Galileo spacecraft the helium abundance in the atmosphere of Jupiter was determined as $Y=m_{\mathrm{He}} /\left(m_{\mathrm{He}}+m_{\mathrm{H}}\right)=0.234$ and was close to the present-day protosolar value $Y=0.275$ [3]. As the model of the Jupiter is significantly determined by its composition and EOS, it was interesting to simulate the thermodynamic properties of the mixture with different compositions in the region of pressure dissociation and ionization.

We considered two mixtures with low and high abundance of helium. The results of calculations for the mixture corresponding to the outer layers of the Jovian atmosphere $(Y=0.234)$ in the region of temperatures from $T=10^{4}$ to $2 \cdot 10^{5} \mathrm{~K}$ and electron number densities from $n_{e}=10^{20}$ to $3 \cdot 10^{24} \mathrm{~cm}^{-3}$ are presented in figure1. The agreement between our calculations and the model [2] along the isotherms $T=4 \cdot 10^{4}, 5 \cdot 10^{4}, 10^{5}$, and $2 \cdot 10^{5} \mathrm{~K}$ is quite good and becomes better with the increase of temperature. The formation of atoms and molecules is the reason of the pressure and energy reduction along the $10^{5} \mathrm{~K}$ isotherm with respect to the isotherm of a non-interacting hydrogen-helium mixture (see figure 10. 

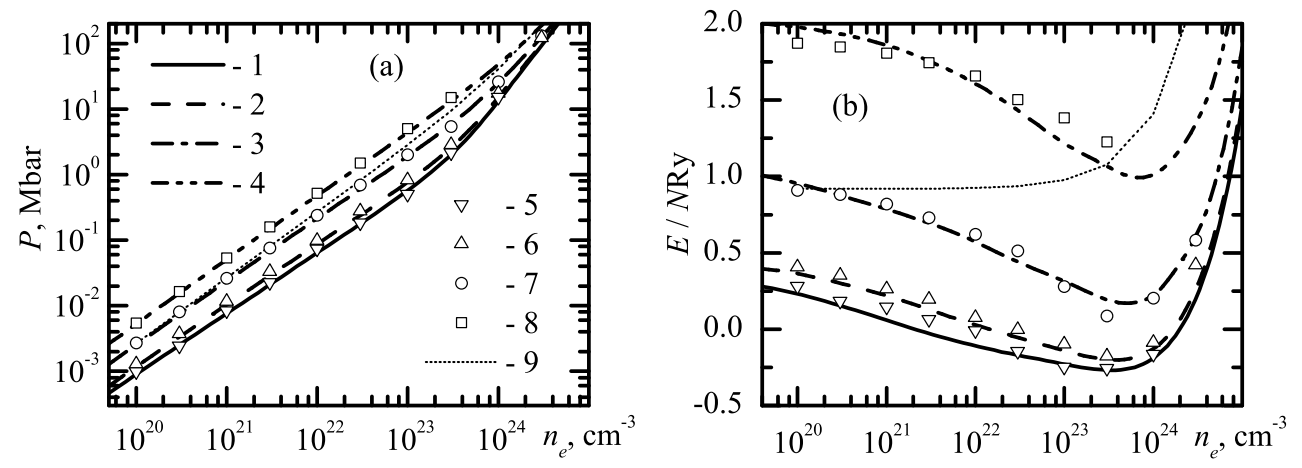

Figure 1. Pressure (a) and energy per particle (b) in a hydrogen-helium mixture with the mass concentration of helium $\mathrm{Y}=0.234(\mathrm{Ry} \approx 13.6 \mathrm{eV})$. Shown are DPIMC isotherms and related EOS isotherms [2]. EOS [2] (DPIMC) calculations: 1(5)-40 kK, $2(6)-50 \mathrm{kK}, 3(7)-100 \mathrm{kK}, 4(8)-200 \mathrm{kK} .9-100 \mathrm{kK}$ isotherm for ideal plasma.
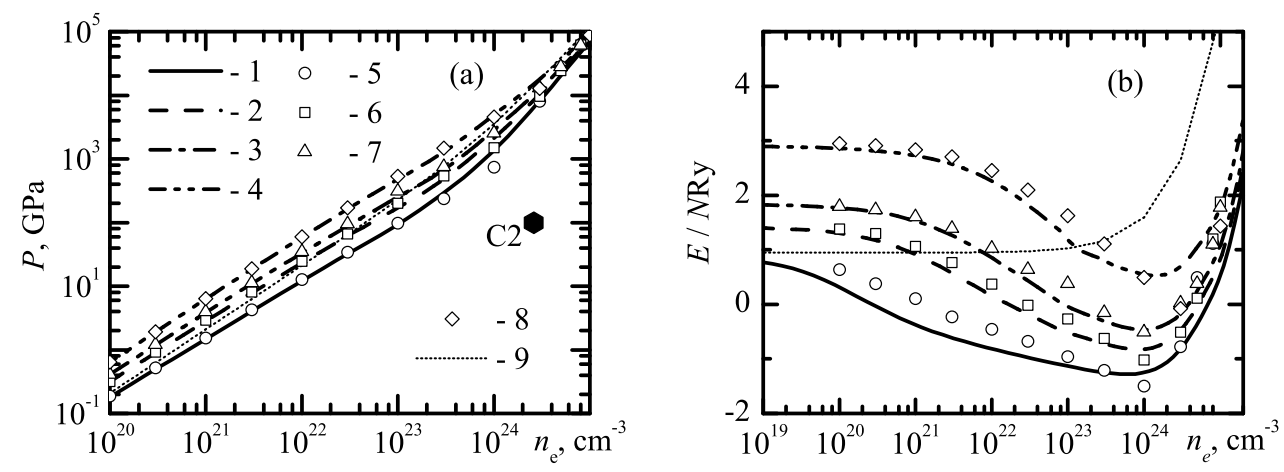

Figure 2. Pressure (a) and energy per particle (b) in a hydrogen-helium mixture with the mass concentration of helium $Y=0.988$. Shown are DPIMC isotherms and related EOS isotherms [2]. EOS [2] (DPIMC) calculations: 1(5) - $100 \mathrm{kK}, 2(6)-$ $156 \mathrm{kK}, 3(7)-200 \mathrm{kK}, 4(8)-312 \mathrm{kK} .9-100 \mathrm{kK}$ isotherm for ideal plasma. C2 critical point of the PPT [8] $\left(T_{c r} \approx 120 \mathrm{kK}\right)$.

The results for $Y=0.988$ (almost pure helium) at relatively high temperatures $T=10^{5} \div 3 \cdot 10^{5} \mathrm{~K}$ in a wide range of densities are presented in figure 2 The agreement between our calculations and the model [2] along the isotherms $T=10^{5}, 1.56 \cdot 10^{5}, 2 \cdot 10^{5}$, and $3.12 \cdot 10^{5} \mathrm{~K}$ is satisfactory for pressure and internal energy per particle. The smaller values of pressure on the DPIMC isotherms $10^{5}$ and $1.56 \cdot 10^{5} \mathrm{~K}$ near the particle density $10^{24} \mathrm{~cm}^{-3}$ can be explained by a strong influence of interaction and bound states in this region (see below). Ionization effects also reduce the internal energy of the system in comparison with non-interacting (ideal) plasma as it can be clearly seen in Fig. 20 b. The positions of ionization minima are well reproduced by the DPIMC method in a good agreement with the chemical picture calculations. At higher densities Fermi-repulsion gives the main contribution to pressure and energy and this effect is also observed in our simulations.

At low temperatures $T<3 \cdot 10^{4} \mathrm{~K}$ and $Y=0.234$ the agreement between 
DPIMC and chemical picture calculations becomes worse, moreover, the region of thermodynamic instability has been discovered. In particular, along the isotherm $T=2 \cdot 10^{4} \mathrm{~K}$ we have found such a region in the range of densities between 0.5 and $5 \mathrm{~g} / \mathrm{cm}^{3}$. Along the isotherms $T=1.5 \cdot 10^{4} \mathrm{~K}$ and $T=10^{4} \mathrm{~K}$ this region is even wider and begins from $0.38 \mathrm{~g} / \mathrm{cm}^{3}$ [12]. Surprisingly, the region of DPIMC instability correlates with the range of temperatures $\left(T<2 \cdot 10^{4} \mathrm{~K}\right)$ and densities $\left(0.3-1 \mathrm{~g} / \mathrm{cm}^{3}\right)$ in which the PPT in hydrogen or hydrogen-helium mixture with low mass concentration has been predicted $[1,8,15]$. Moreover, the sharp rise of electrical conductivity of hydrogenhelium mixture along the quasi-isentrope is also revealed experimentally in the range of densities $0.5-0.83 \mathrm{~g} / \mathrm{cm}^{3}[16]$. However, we cannot claim that these facts confirm the existence of PPT in our DPIMC simulation; in the nearest future we plan to investigate the PPT problem in detail using more sophisticated numerical methods.

Because of the high binding energy of electrons in He we currently can obtain reliable results for $Y=0.988$ only at temperatures higher than $10^{5} \mathrm{~K}$. Under these conditions the influence of helium double ionization can lead to the formation of bound states in the Monte Carlo cell as well as pressure and internal energy decrease. Probably this effect takes place in Fig. 2 near electron number density $n_{e}=10^{24} \mathrm{~cm}^{-3}$ at $T=10^{5}$ and $1.56 \cdot 10^{5} \mathrm{~K}$; the critical point of the possible PPT in this region with critical temperature $\approx 120 \mathrm{kK}[8]$ is also shown in figure [2a.

\section{Deuterium shock Hugoniot}

Using our previous simulation results for deuterium we calculated the shock Hugoniot of liquid deuterium [17]. Figure 3 summarizes the data from different experimental, theoretical, and numerical studies on the shock compression of deuterium. Measurements performed in the NOVA facility, where a shock wave in liquid deuterium with initial density $0.171 \mathrm{~g} / \mathrm{cm}^{3}$ was generated by a laser pulse $[18,19]$ show that the deuterium density behind the shock front can increase by a factor of more than 6 . Experiments with the acceleration of an aluminum foil by a magnetic field to velocities higher than $20 \mathrm{~km} / \mathrm{s}$ [20] show a considerably lower compression ratio in comparison to $[18,19]$. The results obtained in $[18,19]$ and $[20]$ disagree within experimental errors.

In contrast to $[18,19]$ and $[20]$, where targets several hundred microns thick were used, in [21-24], the shock compressibility of solid (initial density $0.199 \mathrm{~g} / \mathrm{cm}^{3}$ ) [21-23] and liquid [23] deuterium was measured in a 4-mm-thick layer using a hemispherical explosive device. It is interesting to note that first such measurements for solid deuterium $[21,23]$ (points 4) showed greater compressibility of deuterium than it was reported later [24] (points 5 in figure 3). The same situation is observed for the experimental points on liquid deuterium (points 6 and 7, correspondingly, see [25] where preliminary experimental data for liquid deuterium from [24] are shown). Experimental points for liquid deuterium [24] are in a good correspondence with the data [20]. Another hemispherical device was applied for shock loading of dense gaseous deuterium with initial density close to that of liquid deuterium [25]. In these experiments [25] apart from 


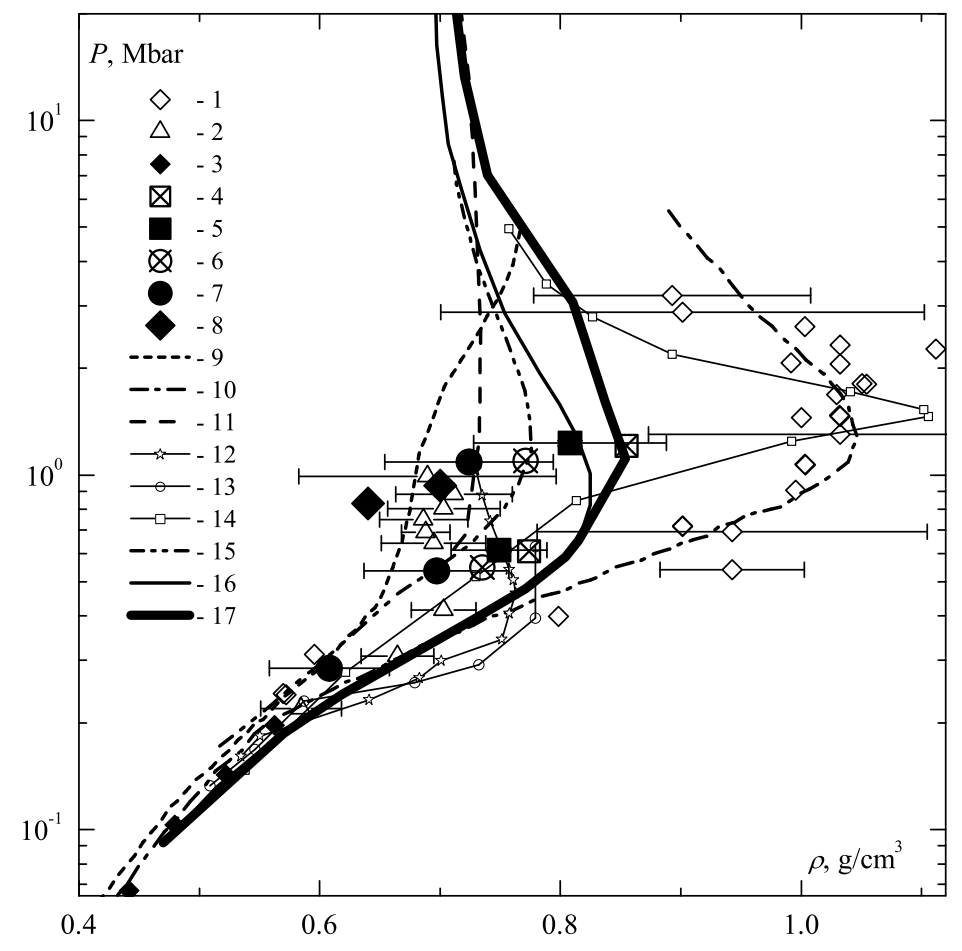

Figure 3. Shock Hugoniot of deuterium. Experimental data for liquid deuterium: $1-$ $[18,19], 2-[20], 3-[26], 6-[25]$, and $7-[24]$; for solid deuterium: $4-[21,23]$, and $5-[24]$; for gaseous deuterium: $8-[25]$. Calculations: $9-[27], 10-[28]$, $11-[29], 12-[30], 13-[31], 14-[32], 15-[25], 16-[33]$, and $17-$ this study.

kinematic shock wave parameters temperature and light absorption of shock-compressed gas were registered. Two experimental points 8 corresponding to the initial gas densities $0.1335 \mathrm{~g} / \mathrm{cm}^{3}$ and $0.153 \mathrm{~g} / \mathrm{cm}^{3}$ are also shown in figure 3 Curve 15 demonstrates the SAHA-IV liquid deuterium Hugoniot with the initial density $0.171 \mathrm{~g} / \mathrm{cm}^{3}$ [25]. The SAHA-IV chemical plasma model was calibrated so as to be in agreement with points 8. In this case curve 15 passes through the old position of the liquid Hugoniot point at 1.09 Mbar [25]. The new position of the point at 1.09 Mbar [24], however, is shifted towards lower densities. Therefore points 7 and 8 in figure 3 probably cannot be described by one and the same theoretical model.

In figure 3 a number of calculated shock Hugoniots is also shown; the detailed analysis of these results can be found in our recent works [17,34]. Here we can only indicate that the DPIMC Hugoniot is shifted towards higher densities in comparison to the experimental data published in $[20,24]$. At pressures below 1-2 Mbar, the thermodynamic instability revealed in [35] comes into play; therefore, a segment of the shock Hugoniot that lies below $1 \mathrm{Mbar}$ is not quite reliable. At higher pressures the closest to the DPIMC Hugoniot is curve 16 calculated in [33] by the classical reactive ensemble Monte Carlo method. In this method, the effects of dissociation of deuterium molecules are taken into account most correctly; this allows one to achieve good 
agreement with the experimental data obtained at low temperatures and pressures [26], even if ionization is not taken into account. Therefore we combined the low-pressure part of the Hugoniot from [33] and high-pressure one from [17] at $15000 \mathrm{~K}$ and obtained the united Hugoniot [34] (curve 17 in figure 31). We want to stress here that these two methods are completely independent and no interpolation procedure is used.

Thus we confirm that the experimental points $[18,19]$ are questionable and the true position of the liquid deuterium Hugoniot remains unclear. We believe that future experiments at the hemispherical device [25] for densities of gaseous deuterium corresponding to the liquid and solid states will give important additional information about shock compression of liquid and solid deuterium. In the nearest future we plan to calculate two DPIMC Hugoniots corresponding to the initial gaseous deuterium densities 0.1335 and $0.153 \mathrm{~g} / \mathrm{cm}^{3}$ from the experiment [25].

\section{Acknowledgments}

This work is supported by the Deutsche Forschungsgemeinschaft via TRR 24, the RAS program No. 17, the CRDF and the Ministry of Education of Russian Federation Grants, and the RF President Grant No. MK-3993.2005.8. The authors are also thankful to the Russian Science Support Foundation.

\section{References}

[1] Chabrier G, Saumon D, Hubbard W B and Lunine J I 1992 Astrophys. J. 391 817-826

[2] Saumon D, Chabrier G and Van Horn H M 1995 Astrophys. J. Suppl. Ser. 99 713-741

[3] Gudkova T V and Zharkov V N 2000 Planet. Space Sci. 47 671-677

[4] Nellis W J 2000 Planet. Space Sci. 48 671-677

[5] Ebeling W, Kraeft W D and Kremp D 1976 Theory of Bound States and Ionization Equilibrium in Plasmas and Solids (Berlin: Akademie-Verlag)

[6] Kraeft W D, Kremp D, Ebeling W and Röpke G 1986 Quantum Statistics of Charged Particle Systems (Berlin: Akademie)

[7] Norman G E and Starostin A N 1968 Sov. Phys. High Temp. 6410

[8] Ebeling W, Förster A, Fortov V E, Gryaznov V K and Polishchuk A Y 1991 Thermophysical Properties of Hot Dense Plasmas (Leipzig: Teubner) ISBN 3-8154-3010-0

[9] Zamalin V M, Norman G E and Filinov V S 1977 The Monte-Carlo Method in Statistical Thermodynamics (Moscow: Nauka)

[10] Filinov V S, Bonitz M, Fortov V E, Ebeling W, Levashov P and Schlanges M 2004 Contrib. Plasma Phys. 44(5-6) 400-406

[11] Filinov V S, Bonitz M, Levashov P R, Fortov V E, Ebeling W, Schlanges M and Koch S W 2003 J. Phys. A.: Math. Gen. 36 6069-6076

[12] Filinov V S, Levashov P R, Bonitz M and Fortov V E 2005 Contrib. Plasma Phys. 45(3-4) 258-265

[13] Filinov V S, Bonitz M and Fortov V E 2000 JETP Letters 72 245-248

[14] Filinov V S, Fortov V E, Bonitz M and Kremp D 2000 Phys. Lett. A 274 228-235

[15] Schlanges M, Bonitz M and Tschttschjan A 1995 Contrib. Plasma Phys. 35 109-125

[16] Ternovoi V Y, Kvitov S V, Pyalling A A, Filimonov A S and Fortov V E 2004 JETP Letters 79(1) $8-11$

[17] Filinov V S, Levashov P R, Bonitz M and Fortov V E 2005 Plasma Phys. Rep. 31(8) 700-704

[18] Da Silva L P, Celliers P et al. 1997 Phys. Rev. Lett. 78 483-486 
[19] Collins G W, Da Silva L B, Celliers P et al. 1998 Science 2811178

[20] Knudson M D, Hanson D L, Bailey J E, Hall C A, Assay J R and Deeney C 2004 Phys. Rev. B 69144209

[21] Belov S I et al 2004 JETP Letters 80(6) 398-404

[22] Belov S I, Boriskov G V et al. 2003 in A L Mikhailov, ed, Substances, Materials and Constructions under Intense Dynamic Influences (Sarov: VNIIEF) pp 100-104

[23] Boriskov G V, Bykov A I, Il'kaev R I, Selemir V D, Simakov G V, Trunin R F, Urlin V D, Fortov V E and Shuikin A N 2003 Doklady Physics 48(10) 553-555

[24] Boriskov G V, Bykov A I, Il'kaev R I, Selemir V D, Simakov G V, Trunin R F, Urlin V D, Shuikin A N and Nellis W J 2005 Phys. Rev. B 71092104

[25] Grishechkin S K et al 2002 JETP Letters 76(7) 433-435

[26] Nellis W J, Mitchell A C, van Theil M, Devine G J, Trainor and R J Brown N 1983 J. Chem. Phys. 791480

[27] 1992 The Los Alamos National Laboratory Equation of State Database (LA-UR-92-3407) (Los Alamos: LANL)

[28] Ross M 1998 Phys. Rev. B 58 669-677

[29] Militzer B and Ceperley D M 2000 Phys. Rev. Lett. 85 1890-1893

[30] Desjarlais M P 2003 Phys. Rev. B 68064204

[31] Bonev S A, Militzer B and Galli G 2004 Phys. Rev. B 69014101

[32] Knaup M, Reinhard P G, Toepferr C and Zwicknagel G 2003 J. Phys. A: Math. Gen. 36(22) $6165-6171$

[33] Bezkrovniy V, Schlanges M, Kremp D and Kraeft W D 2004 Phys. Rev. E 69061204

[34] Bezkrovniy V, Filinov V S, Kremp D, Bonitz M, Schlanges M, Kraeft W D, Levashov P R and Fortov V E 2004 Phys. Rev. E $\mathbf{7 0} 057401$

[35] Filinov V S, Fortov V E, Bonitz M and Levashov P R 2001 JETP Letters 74(7) 384 